\title{
Endobronchial ultrasound: new insight for the diagnosis of sarcoidosis
}

\author{
M. Wong*,\#,§, K. Yasufuku*,\$, T. Nakajima*, F.J.F. Herth`, Y. Sekine*, K. Shibuya*, \\ T. lizasa*, K. Hiroshima ${ }^{+}$, W.K. Lam ${ }^{\#}$ and T. Fujisawa*
}

ABSTRACT: A diagnosis of sarcoidosis should be substantiated by pathological means in order to thoroughly exclude other diseases. The role of real-time endobronchial ultrasound-guided transbronchial needle aspiration (EBUS-TBNA) in the diagnosis of sarcoidosis has not been reported. The purpose of the present study is to evaluate the diagnostic yield of EBUS-TBNA in demonstrating the pathological features of sarcoidosis.

In total, 65 patients with suspected sarcoidosis, with enlarged hilar or mediastinal lymph nodes on computed tomography, were included in the study. Patients with a suspected or known malignancy or previously established diagnosis of sarcoidosis were excluded. Convex probe endobronchial ultrasonography integrated with a separate working channel was used for EBUSTBNA. Surgical methods were performed in those in whom no granulomas were detected by EBUS-TBNA. Patients were followed up clinically.

EBUS-TBNA was performed on a total of 77 lymph node stations in 65 patients. A final diagnosis of sarcoidosis was made for $61(93.8 \%)$ of the patients. The remaining four patients were diagnosed as having Wegener's granulomatosis $(n=1)$ or indefinite $(n=3)$. In patients with a final diagnosis of sarcoidosis, EBUS-TBNA demonstrated noncaseating epithelioid cell granulomas in $56(91.8 \%)$ of the patients. No complications were reported.

Endobronchial ultrasound-guided transbronchial needle aspiration proved to be a safe procedure with a high yield for the diagnoses of sarcoidosis.

KEYWORDS: Bronchoscopy, hilar lymphadenopathy, mediastinum, sarcoidosis, transbronchial needle aspiration, ultrasound

arcoidosis is a multisystemic disorder of unknown aetiology characterised by noncaseating epithelioid cell granulomas. A minority of patients may progress to multiorgan failure. Approximately a quarter of patients with chronic sarcoidosis die due to respiratory failure. The incidence of sarcoidosis has been increasing, possibly explained by greater awareness and recognition of the condition [1]. A diagnosis of sarcoidosis can be greatly substantiated by excluding other disease possibilities, using appropriate clinicoradiological, cytological or histological tissue examination, especially when treatment with systemic steroids is contemplated. Cutaneous involvement occurs in only $\sim 25 \%$ of patients. Erythema nodosum, the hallmark of acute sarcoidosis, is rare in the Japanese [2]. Biopsy of these lesions does not show granulomas [2]. On the contrary, up to $90 \%$ of patients show radiological evidence of thoracic hilar lymph node enlargement and present with acute or insidious respiratory symptoms [3]. Transbronchial lung biopsy (TBLB) is the recommended procedure in most cases. The diagnostic yield, however, depends largely upon the experience of the operator and number of biopsy specimens [2]. Furthermore, TBLB is a procedure that carries a risk of pneumothorax and haemoptysis [4].

Mediastinoscopy has been the method of choice when TBLB is futile $[2,5]$. However, it is invasive, carried out under general anaesthesia, costly, requires in-patient care and has a complication rate of $2-3 \%$ [6]. This realisation led to the search for a less invasive tool with high diagnostic yield and minimal complications.

Convex-probe $(\mathrm{CP})$ endobronchial ultrasonography (EBUS) has thus been evaluated in the present study. A preliminary study using $\mathrm{CP}$ EBUS was performed on surgically resected specimens, and the feasibility of using it to perform real-time EBUS-guided transbronchial needle aspiration (TBNA) was determined prior to its clinical use [7]. EBUS-TBNA was first proven to be clinically useful in the evaluation of mediastinal and hilar lymph nodes under local anaesthesia and conscious sedation [8]. It also played a significant role in the diagnosis and

\section{AFFILIATIONS}

Depts *of Thoracic Surgery, and

+Basic Pathology, Graduate School of Medicine, Chiba University, Chiba, Japan.

"Dept of Medicine, The University of Hong Kong, Queen Mary Hospital, Hong Kong SAR, China.

'Dept of Pneumology and Critical Care Medicine, Thoraxklinik,

University of Heidelberg, Heidelberg, Germany.

${ }^{\S}$ Both authors contributed equally to this manuscript and are considered first co-authors.

\section{CORRESPONDENCE}

T. Fujisawa

Dept of Thoracic Surgery

Graduate School of Medicine

Chiba University

1-8-1 Inohana

Chuo-ku

Chiba 260-8670

Japan

Fax: 81432262172

E-mail: fujisawat@faculty.chiba-u.jp

Received:

February 022006

Accepted after revision:

February 152007

STATEMENT OF INTEREST

None declared. 
staging of lung cancer with hilar and mediastinal lymph node involvement $[9,10]$. To the present authors' knowledge, the role of EBUS-TBNA in the diagnosis of sarcoidosis has not been established. Therefore, the present study was carried out to evaluate the diagnostic yield of EBUS-TBNA in demonstrating granulomas in patients with sarcoidosis.

\section{METHODS}

\section{Patients}

The present study was conducted in both Germany (Chest Clinic, University of Heidelberg, Heidelberg) and Japan (Chiba University, Chiba), between June 2003 and October 2005. All patients with clinical and radiological features suggestive of sarcoidosis were considered if computed tomography (CT) revealed hilar or mediastinal lymph node enlargement (short axis of $>1 \mathrm{~cm}$ ). Patients with a suspected or known malignancy or previously established diagnosis of sarcoidosis were excluded. Written informed consent was obtained from all patients recruited into the study, which was approved by the respective local ethical committee. All patients were managed on an outpatient basis unless already admitted to the hospital for other reasons. Conventional flexible bronchoscopy (model BF-240 bronchovideoscope; Olympus, Tokyo, Japan) was performed first, in a standard fashion, and followed by EBUS-TBNA using the ultrasound bronchoscope (XBFUC260F-OL8; Olympus) on the same bronchoscopy setting. Both bronchoscopic procedures were performed under local anaesthesia and conscious sedation with midazolam in both study centres. In a previous study, EBUS-TBNA of the mediastinal and hilar lymph nodes was shown to be safe and exhibit a good diagnostic yield [8]. The decision as to whether or not to proceed to TBLB, which may give rise to pneumothorax or haemorrhage, was left to the discretion of the operators. A diagnosis of sarcoidosis was made if clinicoradiological findings were supported by pathological tissue demonstrating noncaseating granulomas without necrosis and a negative culture result from EBUS-TBNA, or other surgical methods, such as mediastinoscopy or thoracotomy. Other granulomatous diseases were excluded by reviewing a patient's history and microbiological results. Cases were classified as indefinite if no diagnosis could be made. After the procedures, all patients were followed up clinically and radiologically for 18 months.

\section{Procedure}

CP-EBUS was performed with a convex transducer with a frequency of $7.5 \mathrm{MHz}$ integrated at the tip of a flexible bronchoscope. The outer diameter of the insertion tube of the flexible bronchoscope was $6.7 \mathrm{~mm}$, and that of the tip was $6.9 \mathrm{~mm}$. The angle of view was $90^{\circ}$, and the direction of view was $30^{\circ}$ forward oblique. This CP-EBUS is a linear curvedarray transducer that scans parallel to the insertion direction of the bronchoscope. Images can be obtained by direct contact with the probe or by attaching an inflated balloon filled with saline to the tip, which keeps the probe in contact while sampling the lymph node. The ultrasound image was processed in a dedicated ultrasound scanner (model EUC2000; Olympus) and visualised simultaneously with the conventional bronchoscopy image on the same monitor. This system has an integrated colour Doppler mode which permits blood vessels to be identified and inadvertent puncturing to be avoided. The inner diameter of the working channel was $2.0 \mathrm{~mm}$. A dedicated 22-gauge needle was developed to perform TBNA. The inner diameter of this needle was almost equal to that of a conventional 21-gauge needle, which permits the sampling of histological cores in some cases.

Bronchoscopic procedures were performed orally. The lymph node station was identified according to the International Staging System for Lung Cancer (Mountain classification). The designated lymph node was punctured under direct EBUS guidance. The aspirated material was smeared on to glass slides. Smears were air-dried and fixed in 95\% alcohol. Dried smears were evaluated by an on-site cytopathologist in order to ensure that the cell material obtained was of adequate quality. Adequate cell material was defined as sufficient for a specific diagnosis, such as the presence of noncaseating granulomas without necrosis or the presence of lymphocytes. If adequate tissue was not obtained by on-site cytology after five passes, the procedure was terminated. Furthermore, Papanicolaou staining and light microscopy was carried out by an independent cytopathologist, blinded to case details. Histological specimens were fixed in formalin before being sent to pathology. Aspirated material was also sent for microbiological examination, including special staining for fungi and acid-fast bacilli, as were specimens for culture for tuberculosis and fungi. All patients underwent chest radiography after the procedure to ensure that pneumothorax did not occur.

\section{RESULTS}

In total, 65 patients ( 35 males), with a mean age of $45 \mathrm{yrs}$ (range 19-81), met the inclusion criteria. Both German $(n=42)$ and Japanese $(n=23)$ patients were included in the study population. Of this population, $74 \%$ exhibited radiological stage I disease, whereas the rest had stage II disease. Among all of the lymph nodes with a shortest diameter of $\geqslant 1 \mathrm{~cm}$ on CT, 68 hilar and 134 mediastinal lymph nodes were found matching the typical description of sarcoidosis with bilateral hilar lymphadenopathy. EBUS was able to detect the enlarged lymph nodes in all of the recruited patients, and EBUS-TBNA (fig. 1) was successfully performed in all cases. In all cases, the balloon was used in order to maintain good visualisation during aspiration of the lymph nodes. Granulomas or benign lymphoid cells were detected in the aspirated materials in 62 patients. Of the 65 patients with suspected sarcoidosis, a final diagnosis of sarcoidosis was made in 61 patients (94\%). A total of 77 lymph nodes were recorded and aspirated, giving a mean of 1.2 sampled lymph nodes per patient. This small number of aspirations can be explained by virtue of the rapid on-site cytopathological evaluation; once an adequate sample was obtained, the operator stopped puncturing other lymph nodes (table 1). The mean size of the enlarged lymph nodes, as measured by EBUS, was $20.5 \mathrm{~mm}$ (range 7-37; table 1). Of the enlarged lymph nodes, $67(87 \%)$ were located in the mediastinal region and the remaining $10(13 \%)$ around the hilum or interlobar area. The most commonly sampled lymph node station ( 29 out of $77 ; 38 \%$ ) was subcarinal (station 7 ), where the mean size was $22.4 \mathrm{~mm}$ (range 16-31). More than half (45 out of $77 ; 58 \%$ ) of the enlarged lymph nodes were found in the subcarinal or right lower paratracheal area, located in close proximity to the carina. The largest lymph node $(37 \mathrm{~mm})$ was found in the right-sided hilar area. 

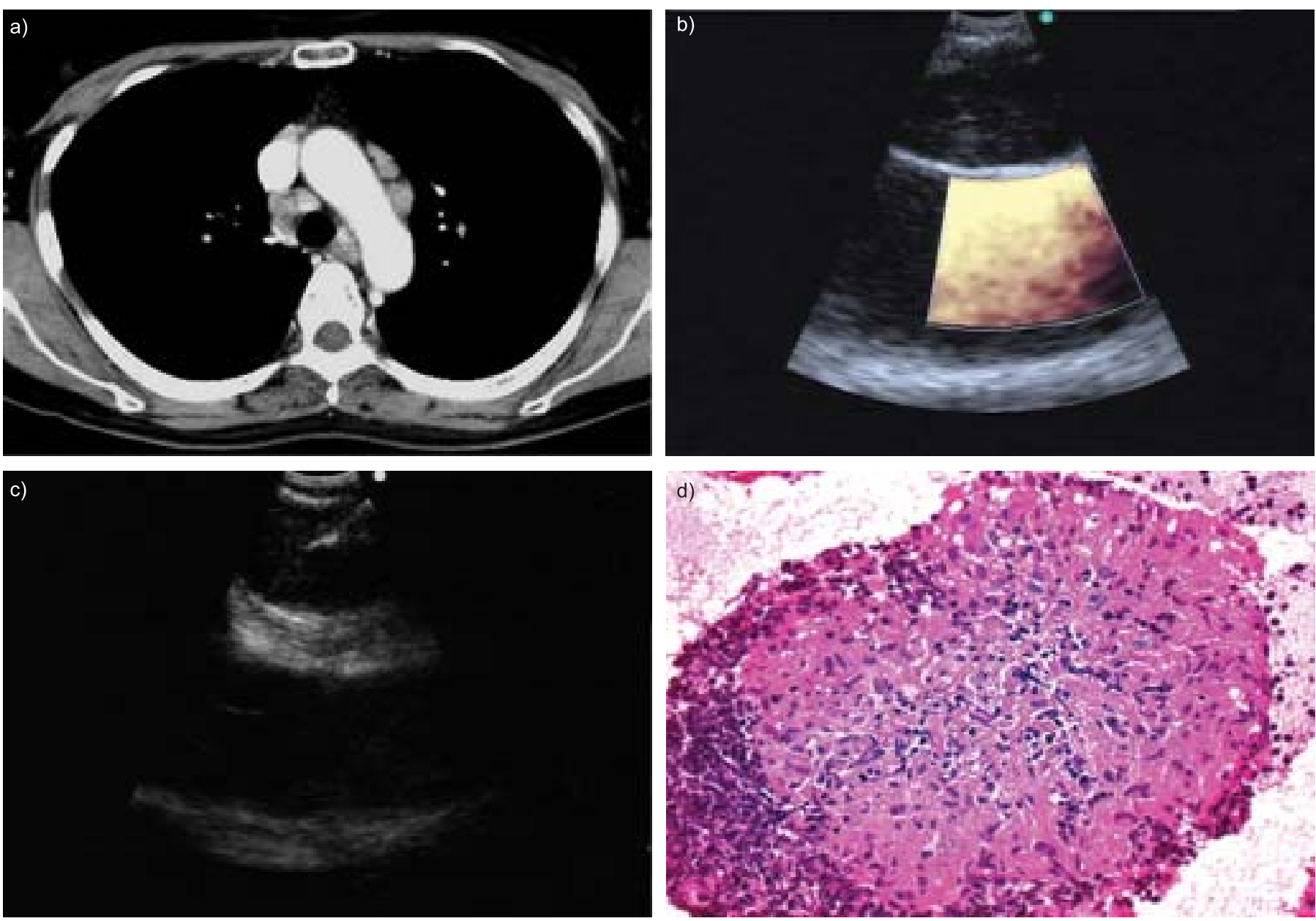

FIGURE 1. a) Computed tomography of the chest showing right lower paratracheal and subaortic lymph node enlargement. b) Endobronchial ultrasonography (EBUS) of the right lower paratracheal lymph node sitting on top of the superior vena cava, confirmed by the colour Doppler signal. c) Convex-probe EBUS-guided transbronchial needle aspiration of the same area, showing the needle within the lymph node. d) Histological specimen, demonstrating a noncaseating granuloma without necrosis, as seen in sarcoidosis.

Inadequate specimens were obtained from three patients. One of them underwent video-assisted thoracoscopic surgery, which showed Wegener's granulomatosis; another underwent mediastinoscopy, which confirmed sarcoidosis; and the final one underwent no further invasive investigation as the patient's condition had been improving (fig. 2). Two patients provided adequate specimens that showed only nonspecific reactive changes. These three patients with indefinite diagnosis were followed up for $\geqslant 18$ months and showed no clinical or radiological deterioration. No patient diagnosed with sarcoidosis in the present trial required amendment of the final diagnosis during follow-up. Microbiological evaluations for tuberculosis or fungal infection all gave negative results.

Among the patients with a final diagnosis of sarcoidosis, EBUS-TBNA was diagnostic in $56(87.5 \%)$ out of 64 patients, assuming that the three patients with indefinite diagnosis also had sarcoidosis. TBLB was performed in 51 (78\%) patients, and the 11 who gave negative TBLB results gave positive EBUS-TBNA results, whereas only two patients gave positive TBLB but negative EBUS-TBNA results. The remaining five patients were confirmed as having sarcoidosis by mediastinoscopy. There were no complications due to pneumothorax, pneumomediastinum or excessive bleeding. Patients were followed up clinically and radiologically over this period.

\section{DISCUSSION}

Pathological specimens are crucial in substantiating a diagnosis of sarcoidosis and excluding other diagnoses, such as tuberculosis, Hodgkin's lymphoma and malignancy, particularly when systemic steroids are contemplated [3]. TBLB is the recommended procedure in most cases [2]. However, it is a procedure with suboptimal yield and a mean diagnostic rate of $65 \%$ (range 40-90) [11, 12], and is also associated with an appreciable complication rate; up to 10 and $5.4 \%$ of patients showed pneumothorax and pulmonary haemorrhage, respectively [4]. Added to this, the realistic diagnostic yield is somewhat lower than reported as the recommended number of biopsy specimens is often not achieved. In the present study, it was shown that the diagnostic yield of EBUS-TBNA reached $87.5 \%$, with no complications noted. This figure takes into account the fact that the three patients classified as being of indefinite diagnosis ultimately had sarcoidosis (i.e. false negatives). The commonest mode of presentation of sarcoidosis is hilar and mediastinal lymphadenopathy, and up to $90 \%$ 


\begin{tabular}{lcc|}
\hline TABLE 1 & $\begin{array}{l}\text { Characteristics of lymph nodes sampled by } \\
\text { endobronchial ultrasound-guided transbronchial } \\
\text { needle aspiration }\end{array}$ \\
\hline Station & Samples & Size mm \\
\hline $\mathbf{2}$ & 11 & $20.5(11-34)$ \\
$\mathbf{2 L}$ & 7 & $16.1(11-21)$ \\
$\mathbf{4 R}$ & 16 & $15.8(7-26)$ \\
$\mathbf{4 L}$ & 4 & $20.3(17-26)$ \\
$\mathbf{7}$ & 29 & $22.4(16-34)$ \\
$\mathbf{1 0 R}$ & 2 & $29.0(19-37)$ \\
$\mathbf{1 0 L}$ & 3 & $19.7(16-23)$ \\
$\mathbf{1 1 R}$ & 4 & $13.0(9-18)$ \\
$\mathbf{1 1 L}$ & 1 & $19.0(19-19)$ \\
Mediastinal & 67 & \\
Hilar or lobar & 10 & $20.5(7-37)$ \\
Total & 77 & \\
\hline
\end{tabular}

Data are presented as $\mathrm{n}$ or mean (range). R: right; L: left.

of patients show evidence of hilar node enlargement on chest radiography [3]. Tissue diagnosis using these areas is, therefore, reasonable, as they are a likely target for confirming the diagnosis, especially since TBLB yielded $30 \%$ of patients with undiagnosed suspected sarcoidosis [13]. The diagnostic yield of TBLB depends upon skill, number of biopsy samples taken and degree of interstitial involvement at the time of biopsy [14-16]. Optimal results can only be achieved if up to 10 biopsy specimens are taken for stage I disease and four or five for stage II disease [11, 17]. The risk of complications increases proportionally with the number of biopsy specimens needed.

TBNA was shown to have a diagnostic yield of $42-76 \%$ for sarcoidosis, with higher yield in stage I disease [18-20]. However, it is performed via blind needle aspiration, guided by prior CT imaging. TBNA is often underutilised, although it is useful in the diagnosis and staging of pulmonary malignancy. In the USA, only $54 \%$ of pulmonologists used TBNA in 2000 , and this low percentage was mainly attributed to the perceived difficulty of this technique [21]. The results of this survey were further confirmed by another trial in which the successful yield of TBNA could only be increased from 21.4 to $47.6 \%$ after a 3-yr period of training [22]. Conversely, the accuracy of EBUS-TBNA in mediastinal staging in lung cancer could be as high as $89 \%$ for the first 20 cases [23]. Information regarding TBNA in the setting of sarcoidosis is not even mentioned as a diagnostic tool in the American Thoracic Society statement on the disease [2].

Mediastinoscopy has a high diagnostic rate and, therefore, has been the procedure of choice when TBLB is futile $[2,5]$. Nonetheless, it is not without limitations. Some intrathoracic lymph nodes (e.g. perihilar lymph nodes, which are typical features of stage I and II sarcoidosis) are inaccessible. In the present study, the largest enlarged lymph node, with a mean size of $29 \mathrm{~mm}$ (range 19-37), was located in the hilar region (table 1), which may provide a better target for needle puncture and subsequently a lower risk of complications. Although only 10 out of 77 (13\%) lymph nodes were located extra-mediastinally, at least some of these patients might require surgical exploration in the absence of EBUS-TBNA.
Furthermore, it is almost impossible to repeat the procedure in the same patient. Generally, sarcoidosis exhibits a particular proclivity for adults aged $<40$ yrs [3], and mediastinoscopy inevitably leaves scars over the neck in these young people.

The recent advances in the endoscopic sampling of mediastinal lymph nodes using EBUS-TBNA and endoscopic ultrasound (EUS)-guided fine needle aspiration (EUS-FNA) have been further developed for the diagnosis and staging of lung cancer or mediastinal lymphadenopathy [13, 23]. With a higher diagnostic yield and minimal complications, EUS-FNA has been used in the diagnosis of mediastinal lymphadenopathy in benign disease. Recent trials of EUS-FNA in sarcoidosis showed a diagnostic value of $82 \%$ [13], sensitivity of $89-100 \%$ and specificity of $94-96 \%$ [24, 25]. Lymphadenopathies in sarcoidosis are typically hilar, and involvement of right paratracheal and aorto-pulmonary window lymph nodes is common (70$76 \%$ ) [26]. EBUS-TBNA is able to sample stations that may be difficult to reach by mediastinoscopy, such as hilar nodes and posterior carinal nodes. Conversely, it has been shown that the right-sided paratracheal and hilar lymph nodes $(2 R, 4 R$ and $10 \mathrm{R})$ are better reached using the transbronchial approach (EBUS) rather than the trans-oesophageal approach (EUS), which can be explained by the fact that the oesophagus is commonly located more to the left of the trachea [27]. This preference for sampling the right-sided paratracheal station has been gauged from the experience of mediastinal lung cancer staging, in which the left paratracheal approach is known to be associated with the worst yields and major complications [28, 29]. Usually, EUS-FNA is incapable of reaching lymph nodes located in the anterior mediastinum and the rest of the thorax beyond the mediastinum. A previous report has revealed difficulty in assessing the fibrotic lymph nodes in stage II

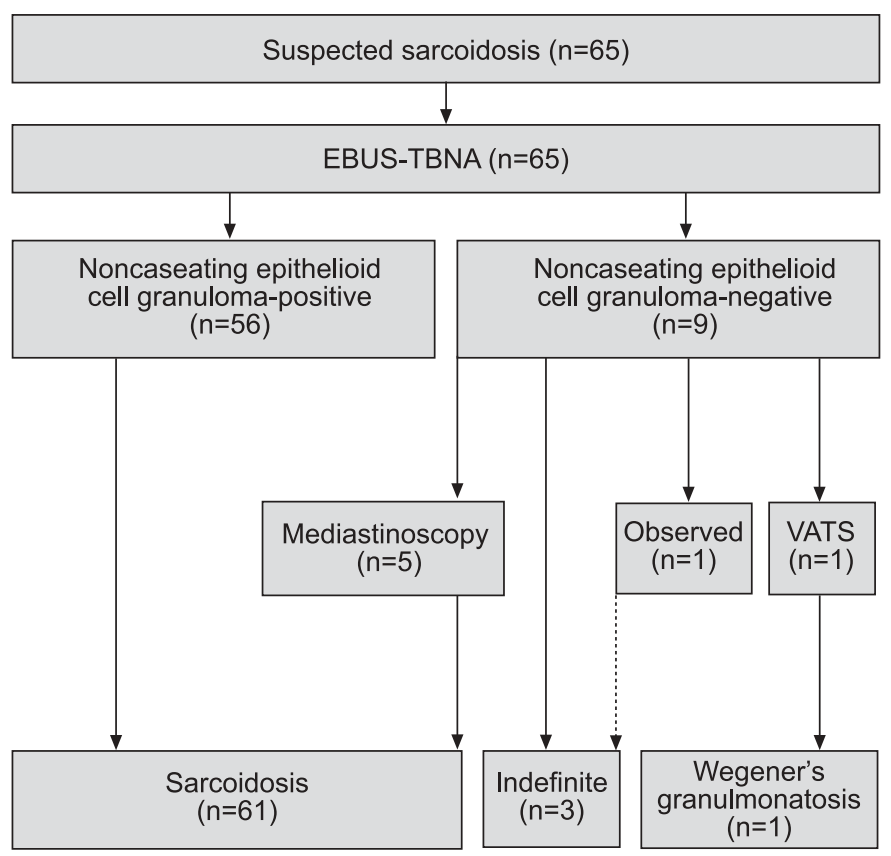

FIGURE 2. Patients suspected of having sarcoidosis were subjected to a diagnostic algorithm. Patients denoted indefinite were followed up but did not receive a specific final diagnosis. EBUS-TBNA: Real-time endobronchial ultrasound-guided transbronchial needle aspiration; VATS: video-assisted thoracoscopic surgery. 
disease using the EUS-FNA approach [13]. However, this problem was not encountered in the present study.

One further advantage of EBUS-TBNA over EUS-FNA lies in the fact that EBUS-TBNA can be performed using conventional bronchoscopy with bronchoalveolar lavage, with or without TBLB, such that peripheral parenchymal, endobronchial and mediastinal lesions can be assessed in the same bronchoscopy setting, without the need for further referral, hence saving time and cost.

The limitations of the present study reflect the inherent nature of sarcoidosis. The high pre-test probability of the disease in the current study population (94\%) could, therefore, have led to bias in this high diagnostic yield. The diagnosis of sarcoidosis can be difficult and relies considerably upon other examinations for the exclusion of other diseases. Tuberculosis is an epidemic disease in Japan, but this situation is confused by the spontaneous remission course of sarcoidosis, since a patient with sarcoidosis may seem to respond to empirical anti-tuberculosis treatment, further confounding the diagnosis of sarcoidosis. As the present study was originally designed to evaluate patients with hilar and/or mediastinal lymph node enlargement, the results obtained here cannot be applied directly to stage III or IV sarcoidosis.

It is envisaged that this accurate, steerable-yet-safe technique may have the potential to serve as an indispensable diagnostic tool in patients suspected of having sarcoidosis, and obviate the need for invasive procedures, with obvious cost-effectiveness implications

\section{REFERENCES}

1 Perng RP, Chen JH, Tsai TT, Hsieh WC. Sarcoidosis among Chinese in Taiwan. J Formos Med Assoc 1997; 96: 697-699.

2 American Thoracic Society, European Respiratory Society. World Association of Sarcoidosis and other Granulomatous Disorders. Statement on sarcoidosis. Am J Respir Crit Care Med 1999; 160: 736-755.

3 Newman LS, Rose CS, Maier LA. Sarcoidosis. N Engl J Med 1997; 336: 1224-1234.

4 McDougall JC, Cortese DA. Bronchoscopic lung biopsy. In: Prakash UBS, ed. Bronchoscopy. New York, Raven Press, 1994; pp. 141-146.

5 Gossot D, Toledo L, Fritsch S, Celerier M. Mediastinoscopy vs thoracoscopy for mediastinal biopsy. Results of a prospective nonrandomized study. Chest 1996; 110: 1328-1331.

6 Hammoud ZT, Anderson RC, Meyers BF, et al. The current role of mediastinoscopy in the evaluation of thoracic disease. J Thorac Cardiovasc Surg 1999; 118: 894-899.

7 Yasufuku K, Chhajed PN, Sekine Y, et al. Endobronchial ultrasound using a new convex probe: a preliminary study on surgically resected specimens. Oncol Rep 2004; 11: 293-296.

8 Yasufuku K, Chiyo M, Sekine Y, et al. Real-time endobronchial ultrasound-guided transbronchial needle aspiration of mediastinal and hilar lymph nodes. Chest 2004; 126: 122-128.

9 Yasufuku K, Chiyo M, Koh E, et al. Endobronchial ultrasound guided transbronchial needle aspiration for staging of lung cancer. Lung Cancer 2005; 50: 347-354.

10 Rintoul RC, Skwarski KM, Murchison JT, Hill A, Walker WS, Penman ID. Endoscopic and endobronchial ultrasound real-time fine-needle aspiration for staging of the mediastinum in lung cancer. Chest 2004; 126: 2020-2022.
11 Gilman MJ, Wang KP. Transbronchial lung biopsy in sarcoidosis. An approach to determine the optimal number of biopsies. Am Rev Respir Dis 1980; 122: 721-724.

12 Koonitz CH, Joyner LR, Nelson RA. Transbronchial lung biopsy via the fiberoptic bronchoscope in sarcoidosis. Ann Intern Med 1976; 85: 64-66.

13 Annema JT, Veselic M, Rabe KF. Endoscopic ultrasoundguided fine-needle aspiration for the diagnosis of sarcoidosis. Eur Respir J 2005; 25: 405-409.

14 Sarcoidosis. In: Seaton A, Seaton D, Leitch AG. Crofton and Douglas's Respiratory Diseases. 4th Edn. Oxford, Blackwell Scientific Publications, 1989; pp. 630-659.

15 Pauli G, Pelletier A, Bohner C, Roeslin N, Warter A, Roegel E. Transbronchial needle aspiration in the diagnosis of sarcoidosis. Chest 1984; 85: 482-484.

16 Morales CF, Patefield AJ, Strollo PJ Jr, Schenk DA. Flexible transbronchial needle aspiration in the diagnosis of sarcoidosis. Chest 1994; 106: 709-711.

17 Roethe RA, Fuller PB, Byrd RB, Hafermann DR. Transbronchoscopic lung biopsy in sarcoidosis. Optimal number and sites for diagnosis. Chest 1980; 77: 400-402.

18 Bilaceroglu S, Perim K, Gunel O, Cagirici U, Buyuksirin M. Combining transbronchial aspiration with endobronchial and transbronchial biopsy in sarcoidosis. Monaldi Arch Chest Dis 1999; 54: 217-223.

19 Trisolini R, Agli LL, Cancellieri A, et al. The value of flexible transbronchial needle aspiration in the diagnosis of stage I sarcoidosis. Chest 2003; 124: 2126-2130.

20 Cetinkaya E, Yildiz P, Altin S, Yilmaz V. Diagnostic value of transbronchial needle aspiration by Wang 22-gauge cytology needle in intrathoracic lymphadenopathy. Chest 2004; 125: 527-531.

21 Colt HG, Prakash UBS, Offord KP. Bronchoscopy in North America: survey by the American Association for Bronchology, 1999. J Bronchol 2000; 7: 8-25.

22 Haponik EF, Cappellari JO, Chin R, et al. Education and experience improve transbronchial needle aspiration performance. Am J Respir Crit Care Med 1995; 151: 1998-2002.

23 Rintoul RC, Skwarski KM, Murchison JT, Wallace WA, Walker WS, Penman ID. Endobronchial and endoscopic ultrasound-guided real-time fine-needle aspiration for mediastinal staging. Eur Respir J 2005; 25: 416-421.

24 Wildi SM, Judson MA, Fraig M, et al. Is endosonography guided fine needle aspiration (EUS-FNA) for sarcoidosis as good as we think? Thorax 2004; 59: 794-799.

25 Fritscher-Ravens A, Sriram PV, Topalidis T, et al. Diagnosing sarcoidosis using endosonography-guided fineneedle aspiration. Chest 2000; 118: 928-935.

26 Bein ME, Putman CE, McLoud TC, Mink JH. A reevaluation of intrathoracic lymphadenopathy sarcoidosis. AJR Am J Roengenol 1978; 131: 409-413.

27 Herth FJ, Lunn W, Eberhardt R, Becker HD, Ernst A. Transbronchial versus transesophageal ultrasound-guided aspiration of enlarged mediastinal lymph nodes. Am J Respir Crit Care Med 2005; 171: 1164-1167.

28 Agli LL, Trisolini R, Burzi M, Patelli M. Mediastinal hematoma following transbronchial needle aspiration. Chest 2002; 122: 1106-1107.

29 Kucera RF, Wolfe GK, Perry ME. Hemomediastinum after transbronchial needle aspiration. Chest 1986; 90: 466. 